\title{
Exploring the Implementation of Artificial Intelligence in the Public Sector: Welcome to the Clerkless Public Offices. Applications in Education.
}

\author{
VASILEIOS YFANTIS ${ }^{1}$, KLIMIS NTALIANIS ${ }^{1}$, FILOTHEOS NTALIANIS ${ }^{2}$ \\ ${ }^{1}$ Department of Business Administration \\ Universityof West Attica \\ Ancient Olive Grove Campus, 250 Thivon\& P. Rallistr, Egaleo 12241, Attica
}

GREECE

${ }^{2}$ Department Of Business Administration
University of Piraeus

Abstract: - The use of the artificial technology in the public administration is currently limited due to several barriers that prevent the decision makers from adopting it. In this paper, we introduce the concept of the clerkless public offices where the concept of the public administration is applied though a synergy of the AI and other technologies aiming at the improvement of the citizen's service even in extreme conditions such as pandemics and physical disasters. The suggested design is an augmentation of the clerkless Amazon Go store and the recent state of the art in e-government. The same Implementation can be held in Education.

Key-Words: -Artificial intelligence, Public administration, E-government, Clerkless, Machine learning, Chatbot. Education, web-based Education, asynchronous Education, synchronous Education.

Received: December 23, 2019. Revised: April 27, 2020. Accepted: May 10, 2020. Published: May 31, 2020.

\section{AI in the public sector}

The digital revolution of the 2000 s created a data orientated world since most of the internet users create records on the net by publishing their data on the social media or other public platforms. The volume of these data increased the need for computing processing services and the creation of the artificial intelligence technology[1]. Artificial Intelligence (AI) is an innovative technology, which has been widely used in the private sector. The advantages of this type of technology are many;however, the most important is its ability to learn a behavior (based on data) and lead to smart decision-making.

The success of the AI in the private sector affected the public sector as well by introducing the AI use in the public administration. The implementation of $\mathrm{AI}$ in the public sector includes critical parts of the public governance such as health, security, education and others [2], [3]. Sousa and other researchers found out that the public sector uses 8 different AI techniques [4] and the most popular is the artificial neutral networks (ANN).The potential benefits from the use of $\mathrm{AI}$ in the public sector are many as well. The more data the AI system retrieves and analyzes the better predictions makes about the public governance. Particularly, the reduction of the cost by using the AI technology, was explored by researchers of the Deloitte University. Eggers and other scholars predicted that the automation of the governmental tasks will save between $\$ 3.3$ billion and $\$ 41.1$ billion [5].According to Mehr who conducted a report in Harvard [6] and the additional research of Androutsopoulou [7] there are 6 governmental issues for which AI is appropriate:

1. Resource allocation

2. Large datasets

3. Experts shortage

4. Predictable scenarios

5. Procedural and repetitive tasks

6. Diverse data aggregation and summarization.

Despite the fact that AI is appropriate as a tool to solve these issues, the adoption and implementation of AI in the public sector is still low. There are several reasons that prevent the political world from implementing the Alin the government, such as investing the public budget in other sectors that could affect the satisfaction of the citizens with the government [8]. Moreover, as Liu and Kim pointed out [9], there still a lack of research about the use of $\mathrm{AI}$ in the public sector.In addition, there is always the challenge for loss of working positions due to the automated tasks. It is obvious that although AI could be a perfect tool for the public sector, the decision makers of the government have to be persuaded about its potentiality. The scope of this 
paper is to persuade the government officials by discussing an innovative use of $\mathrm{AI}$ in the public sector, which might be suitable for most of the governmental issues related with the public services' delivery. This is an adjustment of the clerkless transactions concept [10] for the operation of the public administration. The proposed model is built on a combination of AI technologies that could boost the productivity of the public sector.

\section{The issue of the unavailability of public services}

The level of citizen's service might be limited in terms of quality and quantity for various reasons. For instance during pandemic, physical disasters or other emergent situations, the public servants might be unavailable for work. This situation is very frequent in less developed countries, particularly when the local infrastructure (e.g.roads' condition) is affected by the country's economy. Therefore, the performance of a public servant might not be the ideal one on a daily basis due to health problems that will potentially affect the clerk's ability to be $100 \%$ productive. Other factors include criminality that prevents people from visiting public offices in big cities due to the fear of becoming a victim of a violent action. Although these problems are real, the continuation of the public services even in extreme conditions is critical for the government because it affects the economy. For instance, the unavailability of the municipality's tax clerks due to weather conditions, might affect the level of the daily income for the municipality.

If a local government decides to solve the issue bypunishing the public servants and cutting their wages, it will be a catastrophe. The personnel unions might go on a strike or the servants reduce further their performance as a form of revenge. The suggested solution should be the one with the minimum cost in terms of finance and human resources. Probablya less risky medium such as technology has to be used against this unpleasant situation.

\section{A hybrid AI solution}

The suggested solution is inspired by the culture of the clerkless stores and especially the store of Amazon Go [11]. The clerkless physical store of Amazon opened in 2017 as a grocery store in Seattle, WA. What was different about this store was the fact that it was designed for people who do not like standing in queues and waiting for the cashier to service them. The customers upon entering the store, check in by using a mobile app that downloaded on their smartphone. While they shop inside the store and take the products from the shelf, a sophisticated mix of AI technologies records their moves and recognizes them as buyers of the products. The smart AI system is also able to understand the human moves even if a customer changes his mind and place the product back to the shelf. In this case the product is displayed as still available for other customer to buy it. During the customer's exit from the shop, he just walks out without waiting for a clerk to charge the products he bought. On the contrary, the customer leaves the store and the AI system sends information atthe mobile app about the products that the customer purchased. The mobile app is connected to a credit card or a virtual wallet owned by the customer and he gets charged for the purchased products. For the implementation of this concept, Amazon uses the same technology as self-driving cars [12] including sensors, computer vision and deep learning techniques. The Amazon Go store targets the customers who dislike the queues and prefer an automated way of services dealing only with digital tools. It would be very interesting if we adjust this concept and apply it to the public administration. A clerkless public office would be capable of being available $24 / 7$ by offering services to the citizens and minimizing the cost of the office's operation. However, the design and the business model of the clerkless offices should take into account the local cultures and the level of digital divide among the citizens. For instance, it would be useless to introduce a model of mobile apps to a country, which is digital divided and the citizens do not own smartphones. On the other hand, there is always a way to overcome such barriers by using a less technological advanced solution. Instead of a mobile app, the citizens could use physical smart cards for their transactions inside the Amazon Go "store" of the government.

The design of our solution is citizen orientated and takes into account the citizen's needs. Firstly, a clerkless store of the public sector has to identify the actual reason that the citizen visits the public office. The suggested to tool to detect the citizen's need is an artificial intelligent chatbot, which will lead the citizen to the process. A chatbot is a computer software that uses the natural processing technology (a form of AI technology) to extract information and meaning by receiving an input from a user. The user's input could be text, voice, or other type of input that the machine is able to recognize. For 
instance, face expression is still a way of communication and a machine vision technique will be able to extract information and meaning out of it. The user's input is analyzed by the chatbot to find the intention of the question and then replies to the user with the most suitable answer. The basic architecture of the chatbots includes the 3 main parts:

1. Natural language understanding (NLU)

2. Dialogue manager (DM)

3. Natural language generator (NLG)

Each of the parts plays a specific role in the chatbot. Natural language understanding (NLG) classifies the incoming data from the user and extracts meaning out of it. Particularly, searches is grammar texts, creates patterns and its linguistic output should be appropriate for the dialogue manager. In our case, the user's input will be a question about a public administration task such as tax payment, issuing of a passport, etc. This fact leads to the need for access of the NLG to a database with information related to the public administration.

The next part of the chatbot agent is the dialogue manager, which will receive the data from the NLG. The role of the DM is to prepare the system's responses that will be sent to the natural language generator.The DM adopts a rule based, knowledge-based or retrieval based strategy [13] and gains access to intention and entity templates. Depending on the strategy, it chooses the best template to form a meaningful answer. In case that the user's input does not match any of the templates, the input is analyzed by neural network and online information retrieval components. The neural network components use deep learning/machine learning methods and the online retrieval components look for matching templates online (even on the social networks).

The natural language generator is the last part of the chatbot. The NLG filters the content of the replies and if there are more than 2 correct answers, prioritizes them and displays them based on an internal engagement ranking. The answers are in a text form, however a text to speech tool will transform the text into speech as the final answer. Considering the fact that the citizens might not be familiar with the information communication technologies, speech might be the ideal form of answer.

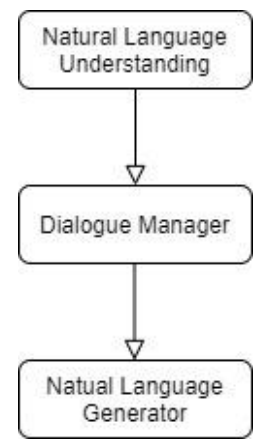

Fig. 1 Data flow in chatbots.
Moreover, if we take the next step to the implementation of our design model, a type of hologram would be a suitable solution to enhance the power of the AI chatbot agent. Hologram is usually an animated 3D image that looks like a human and interacts with other persons. In our case, the chatbot agent will be displayed as a hologram for the citizen user.Actually, the main role of the hologram will be just to make the citizen familiar with a human creature, since the input/output process will be still carried out by the chatbot.

The next step of the process includes the entrance of the citizen to clerkless governmental store depending on the instructions provided by the hologram chatbot. The clerkless store will consist of various rooms and each room will meet the needs of citizen groups. For example, it would be useful for the creation of a room for citizens with disabilities or for a room with more advanced citizens carrying smartphones. The reply of the natural language generator will also include instruction about which room of the clerkless store the citizen will visit.The medium for the entrance could be a smartphone or a smart card (for the less technologically advanced citizens). The following picture depicts the whole process.

Inside the store, which is a collection point for new passports, the passports are placed in shelves just like the products in the Amazon Go store. The process for picking up a new passport is the following:

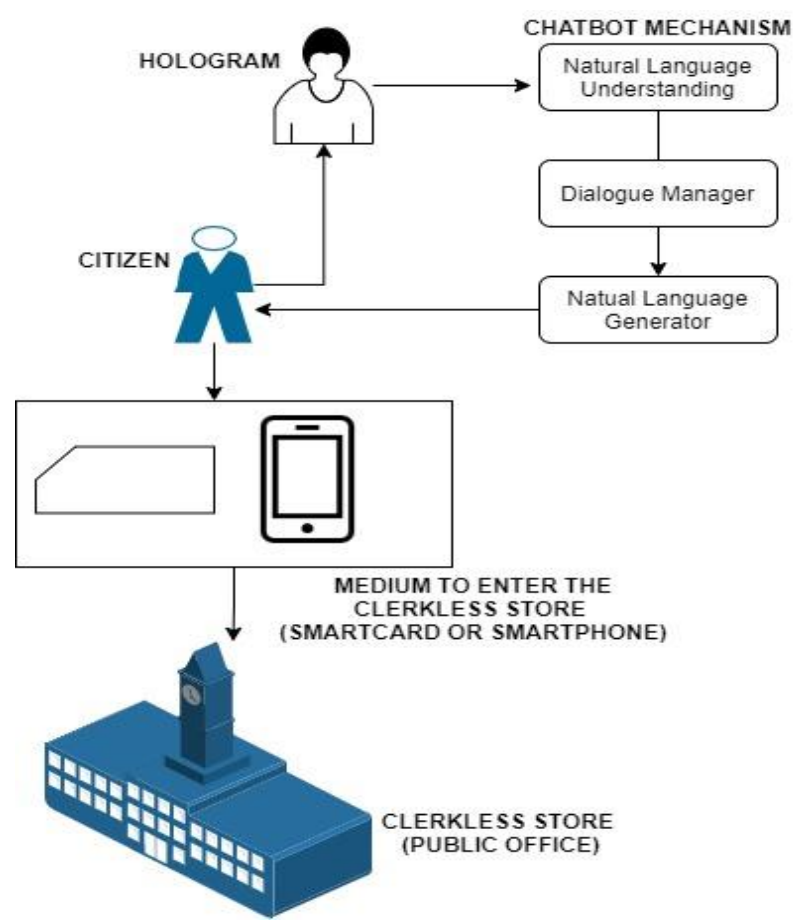

Fig. 2 Chatbot communication and entrance to the clerkless store. 
1. The citizen enters the store and gets authenticated through the government's mobile app or the smart card.

2. The citizen approaches the shelf containing his passport according to the previous instructions by the chatbot.

3. A camera is taking a photo of him when he receives the passport from the shelf. The face recognition technology of the camera matches his biometric information with the information of the new passport.

4. The ctizen picks up the passport from the shelf.

5. The sensor who is placed at the shelf, detects that the passport was received and updates the status of a central system.

6. The citizen checks out on a machine through the mobile app or the smart card and the machine records that the passport was successfully received.

On the backend of the system, deep learning algorithms analyze the citizen's behavior inside the store and train the system based on the learning patterns. For example, useful information for the training of the system includes how much times does the citizen spend in front of the shelves and which shelve is the best position to place the passport.

\section{Conclusion}

$\mathrm{AI}$ is the best tool that the government could use for the public service's delivery due to the power of information included at the citizen's data. A welltrained AI system is capable of detecting the learning patterns and leading to fast decision making. Of course, the implementation of the AI system could lead to the loss of jobs. However, the affected personnel could still keep their jobs if they change a role in the business environment. The public servants could become trainers for the AI system by providing the system with further data about the citizens' service by humans. In this case, they keep their jobs and become trainers of the virtual AI public servants. It is easy to understand that these methodologies can be applied in webbased Education, asynchronous Education and synchronous Education.

\section{References:}

[1] Brynjolfsson, E., McAfee, A., What's driving the Machine Learning explosion?,Harvard Business Review, No 18, 2017, pp. 3-11.

[2] Capmegini, 2017, Unleashing the potential of Artificial Intelligence in the Public Sector, Retrieved from: https://www.capgemini.com/consulting/wp- content/uploads/sites/30/2017/10/ai-in-publicsector.pdf.

[3] Business Insider, 2016, The ATO's virtual assistant has already answered almost a million enquiries this year. Business Insider. Retrieved from https://www.businessinsider.com.au/the-atolaunched-a-siri-for-tax-and-has-called-it-alex-201612.

[4] Gomes de Sousa, W., Pereira De Melo, P., De Souza Bermejo, R., Farias, S. and Oliveira Gomes, A., How and where is artificial intelligence in the public sector going? A literature review and research agenda, Government Information Quarterly, Vol. 36, No 4, 2019, 101392.

[5] Eggers, W., Schatsky, D., Viechnicki, P., 2017, AIaugmented government: Using cognitive technologies to redesign public sector work, Retrieved from https://dupress.deloitte.com/dup-usen/focus/cognitive-technologies/artificialintelligence-government.html.

[6] Mehr, H., 2017, Artificial Intelligence for Citizen Services and Government ArtificialIntelligence for Citizen Services and Government artificial intelligence for citizenservices and government. Retrieved from https://ash.harvard.edu/files/ash/files/artificial_intelli gence_for_citizen_services.pdf.

[7] Androutsopoulou, A., Karacapilidis, N.,Loukis, E., Charalabidis, Y., Transforming the Communication between Citizens and Government through AIguided Chatbots, Government Information Quarterly, Vol. 36, No 2, 2019, pp. 358-367.

[8] Van Deursen, A., Van Dijk, J., Ebbers, W., Why egovernment usage lags behind: Explaining the gap between potential and actual usage of electronic public services in the Netherlands, Electronic Government, 4084 (2006), pp. 269-280.

[9] Liu, S., Kim, Y., Special issue on internet plus government: New opportunities to solve public problems?, Government Information Quarterly, Vol 35, No 1, 2018, pp. 88-97.

[10] Seigel, W., System and method for cashless and clerkless transactions, United States Patent Application Publication, 2003.

[11] Polacco, A. \& Backes, K., The Amazon Go concept: Implications, applications, and sustainability, Journal of Business and Management, Vol. 24, No 1, 2018, pp. 79-92.

[12] Amazon Go Editorial Staff (2020). Amazon Go, Retrieved from https://www.amazon.com/b?node=16008589011.

[13] Moataz M., Mostafa, A., 2020, Chatbot System Architecture, Retrieved from https://wwww.easychair.org/publications/preprint_do wnload/cBTw

\section{Creative Commons Attribution License 4.0 (Attribution 4.0 International, CC BY 4.0)}

This article is published under the terms of the Creative Commons Attribution License 4.0 https://creativecommons.org/licenses/by/4.0/deed.en_US 